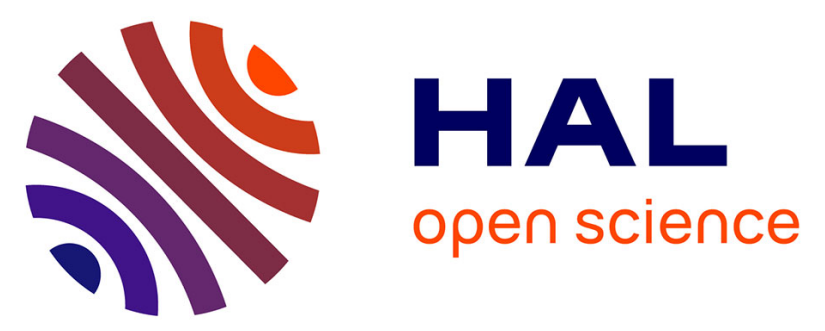

\title{
Disturbed sleep in individuals with idiopathic environmental intolerance attributed to electromagnetic fields (IEI-EMF) : Melatonin assessment as a biological marker
}

Soafara Andrianome, Laurent Hugueville, René de Seze, Maité Hanot-Roy, Kelly Blazy, Christelle Gamez, Brahim Selmaoui

\section{To cite this version:}

Soafara Andrianome, Laurent Hugueville, René de Seze, Maité Hanot-Roy, Kelly Blazy, et al.. Disturbed sleep in individuals with idiopathic environmental intolerance attributed to electromagnetic fields (IEI-EMF) : Melatonin assessment as a biological marker. Bioelectromagnetics, 2016, 37, pp.175182. 10.1002/bem.21965 . ineris-01862939

HAL Id: ineris-01862939

https://hal-ineris.archives-ouvertes.fr/ineris-01862939

Submitted on 28 Aug 2018

HAL is a multi-disciplinary open access archive for the deposit and dissemination of scientific research documents, whether they are published or not. The documents may come from teaching and research institutions in France or abroad, or from public or private research centers.
L'archive ouverte pluridisciplinaire HAL, est destinée au dépôt et à la diffusion de documents scientifiques de niveau recherche, publiés ou non, émanant des établissements d'enseignement et de recherche français ou étrangers, des laboratoires publics ou privés. 
Disturbed sleep in individuals with Idiopathic environmental intolerance attributed to electromagnetic fields (IEI-EMF): melatonin assessment as a biological marker

\author{
Soafara Andrianome ${ }^{1,2}$, Laurent Hugueville ${ }^{3}$, René de Seze ${ }^{1,2}$, Maïté Hanot-Roy ${ }^{1,2}$, Kelly \\ Blazy $^{1,2}$, Christelle Gamez ${ }^{1,2}$, and Brahim Selmaoui ${ }^{1,2}$ \\ ${ }^{1}$ Institut National de l'Environnement Industriel et des Risques (INERIS), Department of \\ Experimental Toxicology, Verneuil-en-Halatte, France \\ ${ }^{2}$ Université de Picardie Jules Verne, Peritox Laboratoire de Périnatalité \& Risques Toxiques \\ UMR-I-01 Unité mixte INERIS, Amiens, France \\ ${ }^{3}$ Centre National de la Recherche Scientifique, Centre MEG-EEG, CRICM et CENIR, UMR 7225, \\ Paris, France \\ Correspondance: Dr Brahim Selmaoui, PhD, HDR \\ Dept. of Experimental Toxicology \\ INERIS, Verneuil-en-Halatte, France \\ E-mail: brahim.selmaoui@ineris.fr
}

Running title: Melatonin assessment in IEI-EMF patients

Conflict of interest: None

Grant sponsor: French National Research Program for Environmental and Occupational Health of ANSES (Projet $n^{\circ}$ 2013-2-02). 


\begin{abstract}
Individuals who suffer from idiopathic environmental intolerance attributed to electromagnetic fields (IEI-EMF) complain of a variety of adverse health effects. Troubled sleep remains a recurrent and common symptom in IEI-EMF individuals. Melatonin, a circadian hormone, plays a major role in the sleep process. In this study, we compared levels of melatonin between a sensitive group (IEI-EMF, $\mathrm{n}=30$ ) and a non-sensitive control group (non IEI-EMF, n=25) without exposure to electromagnetic sources. Three questionnaires were used to evaluate the subjective quality and sleep quantity: the Epworth Sleepiness Scale, the Pittsburgh Sleep Quality Index and the Spiegel Sleep Inventory. Melatonin was quantified in saliva and its major metabolite 6-sulfatoxymelatonin (aMT6s) in urine. Melatonin levels were compared by a two-way analysis of variance at various times between the control and IEI-EMF group. Despite significantly different sleep scores between the two groups, with a lower score in the IEI-EMF group $(p<0.001)$, no statistical difference was found between the two groups for saliva melatonin $(p>0.05)$ and urine aMT6s $(p>0.05)$.
\end{abstract}

Key words: Idiopathic environmental intolerance, Melatonin, Marker, Urine, Saliva, Electromagnetic fields 


\section{Introduction}

During the last decades, the rapid development and extensive use of electric power and wireless communication have increased our exposure to electromagnetic fields (EMF). Potential health effects of EMF are the subject of hot public debate. Moreover, some individuals report adverse effects because of exposure to EMF. This sensitivity is commonly known as idiopathic environmental intolerance of EMF (IEI-EMF) or electromagnetic hypersensitivity (EHS). Among the most common self-reported symptoms of EHS are sleep disorders, headaches, and fatigue [Röösli et al., 2004].

Up until now, numerous double-blind experimental studies have not validated any direct association between the manifestation of these symptoms and EMF exposure [Rubin et al., 2005; Eltiti et al., 2007; Röösli, 2008; Rubin et al., 2009; Kwon et al., 2012] . Individuals claiming EHS did not seem able to detect EMF more accurately than subjects who did not report such hypersensitivity [Eltiti et al., 2007; Röösli, 2008; Rubin et al., 2009] and a generally accepted mechanism of EHS is lacking despite numerous propositions. In addition, there is no known and proven mechanism that triggers EMF-linked symptoms.

Despite lack of association between onset of symptoms and EMF exposure, these symptoms and the suffering of IEI-EMF individuals are real and cannot be ignored.

Recently, we conducted a survey among people self-reporting as suffering from EHS in the French population, and we observed that among reported symptoms, sleep disruption was a recurrent theme and the most common. On the other hand, melatonin ( $N$-acetyl-5methoxytryptamin), a hormone synthesized in the pineal gland with a peak during periods of darkness, has a highly reproducible circadian pattern [Selmaoui and Touitou, 2003] with a 
strong endogenous component and is physiologically controlled by light. This neurohormone seems to play an important role in initiating and maintaining sleep. It was reported that insomnia, sleep deficiency and shorter sleep time in elderly people are associated with the decline of endogenous melatonin concentrations [Lemoine and Zisapel, 2012]. Melatonin administration is effective in restoring normal sleep and improving quality of life in people over 55 years of age [Lemoine and Zisapel, 2012]. In light of the above, we studied whether complaints of sleep disturbances in the IEI-EMF population could be related to low levels of melatonin concentrations.

The aim of the present study was to compare saliva melatonin and urinary 6sulfatoxymelatonin (aMT6s) between a self-declared IEI-EMF group and a non-IEI-EMF group. Saliva and urine samples were collected to measure melatonin and its major metabolite, aMT6s, in urine. We also assessed subjective sleep quality. To our knowledge, this is the first time that melatonin has been assessed in IEI-EMF individuals.

\section{Materials and Methods}

\section{Participants}

This study is part of the survey we conducted in the French IEI-EMF population. Thirty of 52 people reporting as IEI-EMF in France who responded to our questionnaire were recruited for this study ( 7 males and 23 females). The IEI-EMF group included participants whose intolerance duration ranged from 1 to 35 years. Twelve participants also reported multiple chemical sensitivity (MCS) and two reported Lyme's disease. Participants reported sensitivity 
to multiple EMF sources, especially wireless technology (Wi-Fi), cordless phones, cell phones, cell phone base stations and computers.

Patients were from different regions of France and from various self-help groups and associations (local or national) or were independent. Healthy individuals ( $n=25$ with 21 females and 4 males) were matched for gender, age and body mass Index (BMI). They were recruited through Website advertisements and traditional word of mouth or through blogs or online forums or newsgroups.

Inclusion criteria for the IEI-EMF group were participants who reported at least one symptom that they attributed to one or multiple EMF sources and those who had no acute psychiatric disorders such as acute depressive or paranoid psychosis. Exclusion criteria for both groups was the presence of chronic illness. Participants who had consumed antibiotics, anti-inflammatory agents, and melatonin or any other supplements that might interfere with melatonin assessments were also excluded. In addition, shift workers were not admitted to the study. All study participants gave written, informed consent. Protocol was approved by the ethics committee of the CPP Nord Ouest CHU Amiens, France (CPP, 2014/8).

\section{Study design}

Saliva samples were collected with salivettes (Sarstedt, Nümbrecht, Germany). Study subjects collected their two first samples at home to be later delivered to the laboratory for assay. The first sample was collected before bedtime (on day D-1: day before experiment began). The second sample was taken 5 min after they woke up in the morning of experiment day (before coming to laboratory). Participants arrived at the laboratory around 09:00 and spent their waking time in the laboratory. During this period, participants spent 
time inside a dimly lit, electrically shielded room serving as a Faraday's cage. This allowed IEIEMF participants to be in an environment without EMFs. They had a comfortable armchair and were provided with newspapers, magazines, and books. Saliva was then collected every 30 min from 10:00 to $11: 30$ and from $14: 00$ to $16: 30$.

Participants were instructed to abstain from consuming alcohol and coffee for $24 \mathrm{~h}$ before and during each experimental session. They were also advised not to brush their teeth or to do physical exercise for at least $1 \mathrm{~h}$ before sample collection.

Premenopausal women were studied in the laboratory during the follicular phase of their menstrual cycle. During daytime assessment, participants were advised to take a balanced meal. Total urine was collected overnight from 20:00 to wake-up time as night fraction, and from wake-up time to $16: 30$ as daytime fraction (Fig. 1).

\section{Saliva melatonin and urinary aMT6s measurements}

Salivettes were centrifuged ( $2 \mathrm{~min}$ at 3,000 revolutions per $\mathrm{min}$ ) aliquoted and stored at -20 ${ }^{\circ} \mathrm{C}$ until analysis. Melatonin concentration in saliva was determined in duplicate with the ELISA immunoassay technique (Salimetrics, State College, PA). Reported minimum detectable concentration of melatonin for the assay was $1.37 \mathrm{pg} / \mathrm{mL}$. Intra-assay and interassay coefficients of variation were $1.88 \%$ and $10.99 \%$, respectively. Urine samples were stored at $-20{ }^{\circ} \mathrm{C}$ until analysis. Urinary 6-sulfatoxymelatonin was assessed through a competitive ELISA (Buhlmann Laboratories, Schönenbuch, Switzerland) with intra-assay and inter-assay coefficients of variation ranging from $6.99 \%$ and from $12.51 \%$, respectively. The aMT6s concentrations were adjusted for creatinine levels to control for urine volume. Urinary creatinine was measured colorimetrically according to manufacturer's guidelines. 


\section{Subjective assessment of sleep quality}

Subjects completed all surveys on sleep quality. The Pittsburgh Sleep Quality Index (PSQI) [Buysse et al., 1989] measures self-perceived sleep quality during the past month and contains 10 different questions that relate to normal sleep habits. Subjects were asked to respond according to the majority of days and nights in the past month. A global score greater than five corresponds to a "poor sleeper."

The Epworth Sleepiness Scale (ESS) [Johns, 1991] is a self-administered questionnaire for quantitative subjective measurement of sleepiness. For each activity, the patient rates his or her chances of falling asleep during the activity. Scores range from zero (never dozing in a situation) to three (always dozing). Scores for the eight items are summed. Total ESS scores above 10 suggest chronic sleepiness.

The Spiegel Sleep Inventory is a self-administered mini-questionnaire that inquires about the previous night via six questions on sleep initiation, quality, and length; nocturnal awakenings; dreams; and feeling refreshed in the morning. A score below 15 suggests pathologic sleep, whereas a score above 20 is considered good sleep [Spiegel, 1981].

\section{Statistical analysis}

Comparisons between control and IEI-EMF groups were performed with U Mann Whitney for sleep evaluation of PSQI, Spiegel and Epworth scores; two-way analysis of variance (ANOVA) for testing time and groups effect considering saliva melatonin or urinary aMT6s as variables. For characteristics and sleep scores comparisons, the student t-test and chi square test were used. 
Statistical analysis was performed with the help of SPSS statistical software (IBM SPSS Statistics for Windows, Version 20.0. Armonk, NY) and plotted with the Graphpad Prism (Prism, version 5, San Diego, CA). Values were considered to be significantly different when $p$ $<0.05$.

\section{Results}

The characteristics of the two groups are presented in Table 1. Groups did not differ statistically in age, gender and smoker proportions. The IEI-EMF group reported significantly different scores from the control group, showing a lower quantity $(p<0.001)$ and quality $(p<0.001)$ of sleep. The ESS was similar for both groups $(p=0.183)$. Additionally, the IEI-EMF group had a significantly higher proportion of participants with Spiegel score under the cutoff value of $15(p<0.01)$.

Figure $2 \mathrm{a}$ represents data of 6-sulfatoxymelatonin concentrations in urine (aMT6s) and Figure $2 \mathrm{~b}$ those of aMT6s adjusted by urinary creatinine concentrations. Two-way ANOVA performed for both sets of data did not reveal any differences between aMT6s levels measured in the IEI-EMF group and those in the control ( $p>0.05)$. However, time effect was found to be very significant $(p<0.0001)$ for aMT6s levels between daytime and nighttime fractions.

Figure 3 represents data of saliva melatonin assessed at different times of day: bedtime, wake-up time, then subsequently every 30 min between 10:00 and 11:30 and between 14:00 and 16:30. Here again, no significant differences ( $p>0.05)$ were shown between saliva melatonin concentrations in the IEI-EMF group and those obtained in the control. Participants noted their bedtime and wake-up times (Table 2). 


\section{Discussion}

The present work was intended to determine whether melatonin levels are affected in patients suffering from IEI-EMF. In fact, IEI-EMF patients complained about insomnia or difficulties in sleeping and headache disorders. On the other hand, lower nocturnal melatonin levels were reported to be associated with worsened daytime sleepiness, sleep deficiency, and shorter sleep time in older men [Lemoine and Zisapel, 2012]. Moreover, a strong association has also been reported between melatonin secretion and headache disorders. Indeed, some studies suggest that some people who have migraine might be deficient in melatonin production [Bruera et al., 2008; Masruha et al., 2010].

Our results showed that urinary aMT6s levels were not affected in IEI-EMF patients compared with non-IEI-EMF individuals. Despite poor sleep quality reported by IEI-EMF patients (PSQI index) and the high proportion of IEI-EMF presenting a pathologic Spiegel score, their day/night levels of urinary melatonin were comparable to those of non-IEI-EMF individuals. In addition, day/night variations of melatonin levels were in accordance with what is observed in normal circadian rhythm, with high levels during the night and low levels during the day. This suggests that circadian rhythm in IEI-EMF patients is not affected.

In addition, saliva melatonin was assessed in a sample collected just before bedtime and analyzing melatonin in this sample could be an indicator for melatonin onset. Indeed, the onset of melatonin secretion is associated with an increase in sleep propensity in both sighted and blind individuals [Lewy et al., 1980; Lockley et al., 1997; Wyatt et al., 2006] and it was reported that exogenous administration of melatonin especially during the day can 
facilitate sleep [Dollins et al., 1994; Cajochen et al., 1996; Zhdanova et al., 1996; Hughes and Badia, 1997; Stone et al., 2000; Rajaratnam et al., 2004; Wyatt et al., 2006; Arendt et al., 2008].

In our present work, saliva melatonin concentrations at bedtime did not differ between the IEI-EMF group and the control. This means that melatonin levels before bedtime were similar in both groups. Moreover, saliva melatonin concentrations collected immediately after wake-up time and in subsequent samples collected every $30 \mathrm{~min}$ from 10.00 to 16.30 did not reveal any changes in daytime pattern of melatonin secretion. These results suggest therefore that the circadian rhythm of melatonin secretion is most likely not phase-shifted in IEI-EMF patients.

However, the best way to evaluate the phase shift of melatonin circadian rhythm would be a temporal sampling over $24 \mathrm{~h}$ or at least during the whole night to include melatonin peak secretion. Nevertheless, our protocol combining day/night variation of melatonin levels in urine and saliva melatonin analyzed in samples collected at specific moments of the day, such as before bedtime and immediately after wake-up time, remains quite informative about the circadian rhythm of melatonin phase shifting.

Although chronotype profile was not investigated by an appropriate questionnaire, our results indicate an earlier waking time in the IEI-EMF group despite comparability to the control group in terms of age and gender. The bedtimes (or wake-up times) differed between the two groups by about $1 \mathrm{~h}$. This difference in timing of the sleep/wake schedule cannot be described in terms of chronotype. Indeed, evening chronotypes typically have time of sleep delayed 2 to $3 \mathrm{~h}$ longer than morning chronotypes [Lack et al., 2009]. In terms of circadian markers, our study did not find any significant differences between IEI-EMF and 
control groups in the timing of saliva melatonin levels analyzed at bedtime, wake-up time and in subsequent samples. It should be noted that the chronotype depends also on environmental [Roenneberg et al., 2003] and genetic [Toh et al., 2001] factors.

The main limitation of this study is the variable time of sampling in participants' homes. Although we used a largely acceptable form of sampling for saliva collection and despite participants being instructed to collect samples in a given time range, variations in time of collection at home were unavoidable. However, sampling was performed so as not to disrupt participant habits and to reduce stress. Home collections concern only a small proportion of study samples.

Our hypothesis in studying melatonin in IEI-EMF individuals is based on the fact that its secretion is strongly inhibited by light [Lewy et al., 1980], which is the visible portion of EMFs. Therefore it is speculated that melatonin could likely be sensitive to another part of the electromagnetic spectrum. With regard to extremely low-frequency magnetic fields (1 to $300 \mathrm{~Hz}$ ), data from the literature on the effect of EMFs on melatonin secretion are contradictory in animal studies [Touitou and Selmaoui, 2012]. On the other hand, the circadian pattern of the hormone can be phase-shifted (advanced or delayed) by light according to the time of exposure [Touitou $Y$ et al., 1993], and this phenomenon might occur with exposure to EMFs and could in part explain the poor sleep quality in IEI-EMF patients.

Data showed no differences between melatonin concentrations measured in the IEI-EMF group and those measured in the control. In light of these results, it is suggested that melatonin was not affected in our group of IEI-EMF patients and cannot be related to their sleep disturbance. Likewise, it is not excluded that perception of sleep instead of sleep can be affected. While sleep quality measured by self-report questionnaire is suitable, it can be 
argued that time taken to fall asleep and/or number of sleeping hours could be more precisely assessed with physiological measures.

Origins of this sleep disruption or eventual troubled perception of sleep should also be investigated more precisely.

\section{Conclusions}

The present work was conducted to determine whether IEI-EMF patients have low levels of melatonin that might explain their poor sleep.

However, these results must be interpreted with caution. Absence of a correlation between sleep complaints and melatonin levels does not mean that IEI-EMF patients are not really suffering from sleep disturbance. In view of this, polysomnographic recordings are an effective way to evaluate sleep disturbances objectively. In addition, the origin of these problems must be sought as well as EMF's role in occurrence of symptoms.

Additionally, some IEI-EMF patients have reported to us that symptoms such as headache, itching, etc. occur several hours after exposure and might appear at bedtime. Headache pain and so on could likely be the cause of sleep disturbance. Indeed, some studies have shown that more than $50 \%$ of patients with diverse sources of chronic pain complain significantly of sleep disturbance [Pilowsky et al., 1985; Atkinson et al., 1988 ; Smith et al., 2000].

Overall, there are very few data available on IEI-EMF's biomarkers [Dahmen et al., 2009; Ghezel-Ahmadi et al., 2010]. Further studies are needed to look for other biomarkers that may help in diagnosing IEI-EMF.

\section{Acknowledgments}


This work is part of the PhD thesis of Soafara Andrianome. We would like to thank all participants in the study.

\section{References:}

Arendt J, Bojkowski C, Folkard S, Franey C, Marks V, Minors D, Waterhouse JC, Wever RA, Wildgruber C, Wright J. 2008. Some Effects of Melatonin and the Control of its Secretion in Humans. In: Evered, D, Clark, S, editors. Novartis Found Symp Chichester, UK: John Wiley \& Sons. pp. 266283.

Atkinson JH, Ancoli-Israel S, Slater MA Garfin SR, Gillin JC. 1988 Subjective sleep disturbance in chronic back pain. Clin J Pain 65:225-232.

Bruera O, Sances G, Leston J, Levin G, Cristina S, Medina C, Barontini M, Nappi G, Figuerola MAA de L. 2008. Plasma melatonin pattern in chronic and episodic headaches: evaluation during sleep and waking. Funct Neurol 23:77-81.

Buysse DJ, Reynolds CF, Monk TH, Berman SR, Kupfer DJ. 1989. The Pittsburgh Sleep Quality Index: a new instrument for psychiatric practice and research. Psychiatry Res 28:193-213.

Cajochen C, Kräuchi K, Arx MA von, Möri D, Graw P, Wirz-Justice A. 1996. Daytime melatonin administration enhances sleepiness and theta/alpha activity in the waking EEG. Neurosci Lett 207:209-213.

Dahmen N, Ghezel-Ahmadi D, Engel A. 2009. Blood laboratory findings in patients suffering from selfperceived electromagnetic hypersensitivity (EHS). Bioelectromagnetics 30:299-306.

Dollins AB, Zhdanova IV, Wurtman RJ, Lynch HJ, Deng MH. 1994. Effect of inducing nocturnal serum melatonin concentrations in daytime on sleep, mood, body temperature, and performance. Proc Natl Acad Sci U S A 91:1824-1828.

Eltiti S, Wallace D, Ridgewell A, Zougkou K, Russo R, Sepulveda F, Mirshekar-Syahkal D, Rasor P, Deeble R, Fox E. 2007. Does short-term exposure to mobile phone base station signals 
increase symptoms in individuals who report sensitivity to electromagnetic fields? A doubleblind randomized provocation study. Environ Health Perspect 115:1603-1608.

Ghezel-Ahmadi D, Engel A, Weidemann J, Budnik LT, Baur X, Frick U, Hauser S, Dahmen N. 2010. Heavy metal exposure in patients suffering from electromagnetic hypersensitivity. Sci Total Environ 408:774-778.

Hughes RJ, Badia P. 1997. Sleep-promoting and hypothermic effects of daytime melatonin administration in humans. Sleep 20:124-131.

Johns MW. 1991. A new method for measuring daytime sleepiness: the Epworth sleepiness scale. Sleep 14:540-545

Kwon MK, Choi JY, Kim SK, Yoo TK, Kim DW. 2012. Effects of radiation emitted by WCDMA mobile phones on electromagnetic hypersensitive subjects. Environ Health Glob Access Sci Source 11:69.

Lack L, Bailey M, Lovato N, Wright H. 2009. Chronotype differences in circadian rhythms of temperature, melatonin, and sleepiness as measured in a modified constant routine protocol. Nat Sci Sleep 1:1-8.

Lemoine P, Zisapel N. 2012. Prolonged-release formulation of melatonin (Circadin) for the treatment of insomnia. Expert Opin Pharmacother 13:895-905.

Lewy AJ, Wehr TA, Goodwin FK, Newsome DA, Markey SP. 1980. Light suppresses melatonin secretion in humans. Science 210:1267-1269.

Lockley SW, Skene DJ, Tabandeh H, Bird AC, Defrance R, Arendt J. 1997. Relationship between napping and melatonin in the blind. J Biol Rhythms 12:16-25.

Masruha MR, Lin J, de Souza Vieira DS, Minett TSC, Cipolla-Neto J, Zukerman E, Vilanova LCP, Peres MFP. 2010. Urinary 6-sulphatoxymelatonin levels are depressed in chronic migraine and several comorbidities. Headache 50:413-419.

Pilowsky I, Crettenden I, Townley M. 1985. Sleep disturbance in pain clinic patients. Pain 23:27-33. 
Rajaratnam SMW, Middleton B, Stone BM, Arendt J, Dijk DJ. 2004. Melatonin advances the circadian timing of EEG sleep and directly facilitates sleep without altering its duration in extended sleep opportunities in humans. J Physiol 561:339-351.

Roenneberg T, Wirz-Justice A, Merrow M. 2003. Life between clocks: daily temporal patterns of human chronotypes. J Biol Rhythms 18:80-90.

Röösli M. 2008. Radiofrequency electromagnetic field exposure and non-specific symptoms of ill health: a systematic review. Environ Res 107:277-287.

Röösli M, Moser M, Baldinini Y, Meier M, Braun-Fahrländer C. 2004. Symptoms of ill health ascribed to electromagnetic field exposure--a questionnaire survey. Int J Hyg Environ Health 207:141150.

Rubin GJ, Munshi J Das, Wessely S. 2005. Electromagnetic hypersensitivity: a systematic review of provocation studies. Psychosom Med 67:224-232.

Rubin GJ, Nieto-Hernandez R, Wessely S. 2009. Idiopathic environmental intolerance attributed to electromagnetic fields (formerly "electromagnetic hypersensitivity"): An updated systematic review of provocation studies. Bioelectromagnetics:31:1-11

Selmaoui B, Touitou Y. 2003. Reproducibility of the circadian rhythms of serum cortisol and melatonin in healthy subjects: a study of three different 24-h cycles over six weeks. Life Sci 73:3339-3349.

Smith MT, Perlis ML, Smith MS, Giles DE, Carmody TP. 2000. Sleep quality and presleep arousal in chronic pain. J Behav Med 23:1-13.

Spiegel R. 1981. Sleep and sleepiness in advanced age. In: Weitzman ED ed. Advances in Sleep Research, vol 5. Jamaica, NY: Spectrum Publications, MTP Press. pp. 272.

Stone BM, Turner C, Mills SL, Nicholson AN. 2000. Hypnotic activity of melatonin. Sleep 23:663-669.

Toh KL, Jones CR, He Y, Eide EJ, Hinz WA, Virshup DM, Ptácek L, Fu YH. 2001. An hPer2 phosphorylation site mutation in familial advanced sleep phase syndrome. Science 291:1040-1043. 
Touitou Y, Arendt J, Pevet P. 1993. Melatonin and the Pineal Gland: from Basic Science to Clinical Applications. Amsterdam, the Netherlands: Elsevier. pp. 141-147

Touitou Y, Selmaoui B. 2012. The effects of extremely low-frequency magnetic fields on melatonin and cortisol, two marker rhythms of the circadian system. Dialogues Clin Neurosci 14:381399.

Wyatt JK, Dijk DJ, Ritz-de Cecco A, Ronda JM, Czeisler CA. 2006. Sleep-facilitating effect of exogenous melatonin in healthy young men and women is circadian-phase dependent. Sleep 29:609618.

Zhdanova IV, Wurtman RJ, Morabito C, Piotrovska VR, Lynch HJ. 1996. Effects of low oral doses of melatonin, given 2-4 hours before habitual bedtime, on sleep in normal young humans. Sleep 19:423-431. 


\section{Bioelectromagnetics}

Figure and table legends

Figure 1. Description of protocol: saliva and urine collection

Urine collection was fractioned (1): night fraction: from 20:00 (D-1) to wake-up on D; (2): daytime fraction: from participant's arrival to $16: 30$ on D.

Saliva collection: on day D-1: before sleeping; on day D: 5 min after wake-up, and every 30 min from 10:00 to $11: 30$ and from $14: 00$ to $16: 30$.

D-1: day before experiment; D: day of experiment

Figure 2. Day/night variation of 6-sulfatoxymelatonin concentrations in urine.

a) aMT6s concentrations, no significant difference between control (white bars) and IEI-EMF group (black bars).

b) Creatinine-adjusted 6-sulfatoxymelatonin, no significant difference between control (white bars) and IEI-EMF group (black bars). Data represent the mean \pm S.E.M. of concentrations in control $(n=25)$ and IEI-EMF $(n=30)$ group.

Figure 3. Saliva melatonin concentrations $(\mathrm{pg} / \mathrm{mL})$ in samples collected at bedtime, waking time and every $30 \mathrm{~min}$ from $10: 00$ to $11: 30$ and from $14: 00$ to $16: 30$. No significant difference between control (white bars) and IEI-EMF (black bars) group. Data represent mean \pm S.E.M. Control $(n=25)$ and IEI-EMF $(n=30)$.

Table 1. Participants' characteristic and sleep scores. Information presented as mean \pm SD or total number $\mathrm{n}$ (percentage). 
Table 2. Intervals, mean and median of bedtimes and wake-up time of participants collected during and before day of experimentation (D-1). 


\section{Bioelectromagnetics}

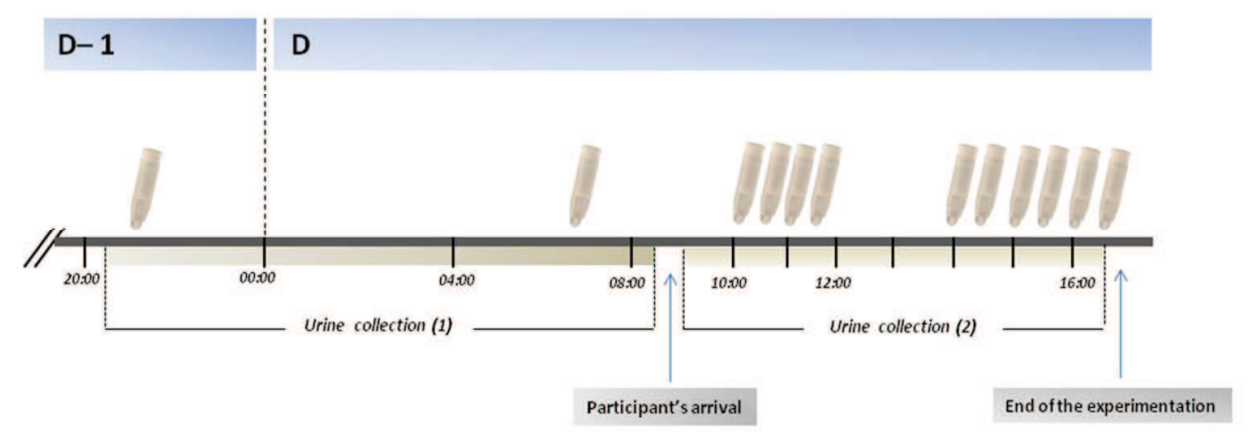

$254 \times 190 \mathrm{~mm}(96 \times 96 \mathrm{DPI})$

John Wiley \& Sons 

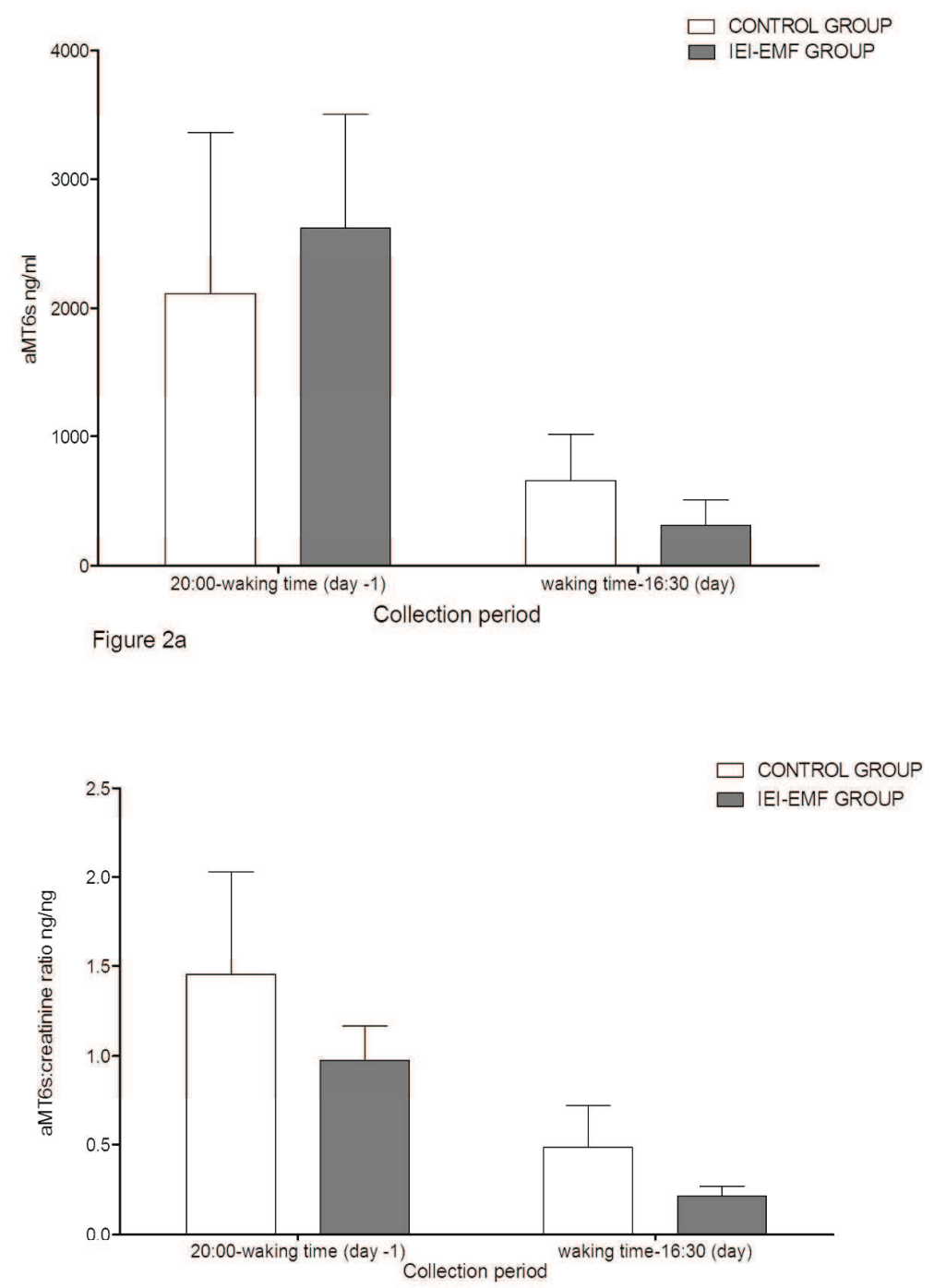

Figure $2 b$

$149 \times 212 \mathrm{~mm}(300 \times 300$ DPI $)$ 


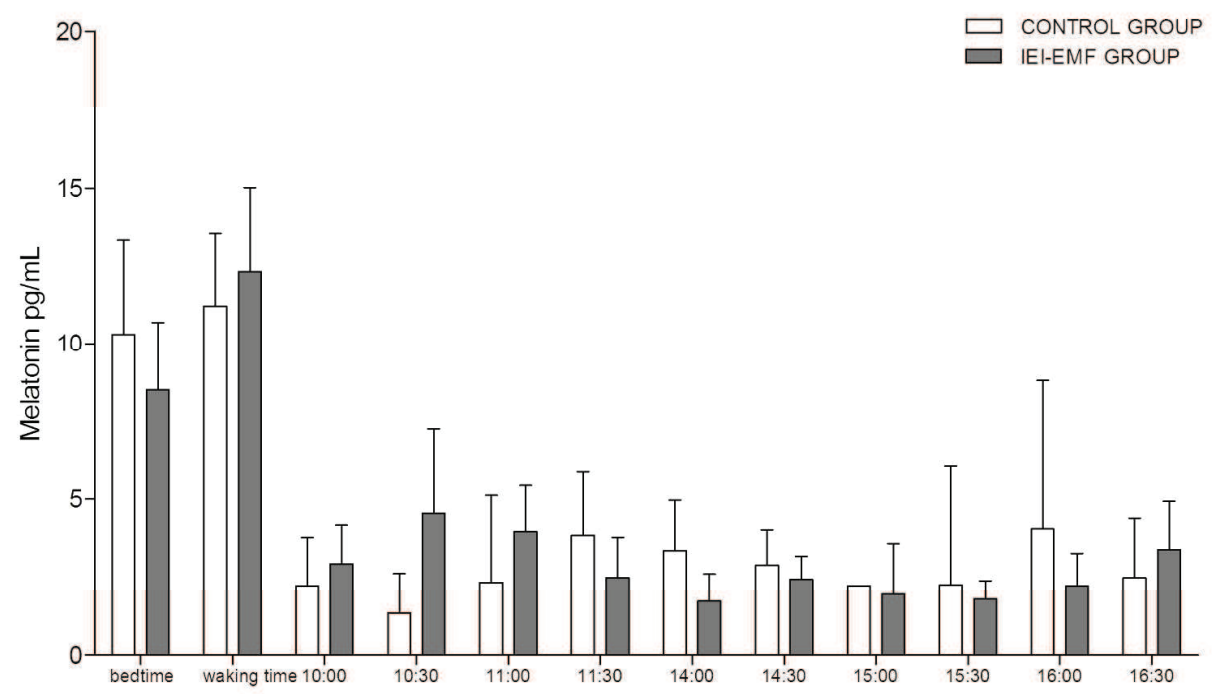

$196 \times 112 \mathrm{~mm}(300 \times 300$ DPI $)$ 
Table 1. Participants' characteristic and sleep scores. Data presented as mean \pm S.D. or total number $\mathrm{n}$ (percentage)

\begin{tabular}{|c|c|c|c|}
\hline Variable & $\begin{array}{l}\text { Control group } \\
(n=25)\end{array}$ & $\begin{array}{l}\text { IEI-EMF group } \\
(n=30)\end{array}$ & p-value \\
\hline Age, years & $46 \pm 10$ & $47 \pm 9$ & 0.999 \\
\hline IEI-EMF duration, years & - & $8.43 \pm 7.3$ & - \\
\hline Female gender, $\mathrm{n}(\%)$ & $21(84)$ & $23(77)$ & 0.498 \\
\hline $\mathrm{BMI}, \mathrm{kg} / \mathrm{m}^{2}$ & $22.80 \pm 2.60$ & $22.35 \pm 3.33$ & 0.268 \\
\hline Smoker $\mathrm{n}(\%)$ & $6(24)$ & $4(13)$ & 0.307 \\
\hline Spiegel score & $21 \pm 6$ & $16 \pm 6$ & $<0.001$ \\
\hline Spiegel score <15, n (\%) & $8(27)$ & $21(70)$ & $<0.01$ \\
\hline ESS & $7 \pm 4$ & $9 \pm 4$ & 0.183 \\
\hline ESS $>10, n(\%)$ & $7(28)$ & $12(40)$ & 0.404 \\
\hline PSQI index & $3 \pm 2$ & $8 \pm 3$ & $<0.001$ \\
\hline PSQI >5, n (\%) & $1(4)$ & $5(20)$ & 0.134 \\
\hline
\end{tabular}

Abbreviations: IEI-EMF: Idiopathic environmental Intolerance attributed to electromagnetic fields; BMI: body mass Index, ESS: Epworth Sleep Score PSQI: Pittsburgh Sleep Quality Index Respective cutoffs for sleep scores were: Spiegel score under 15 ; PSQI above 5 and ESS above 10 
Table 2. Intervals, mean and median of bedtimes and wake-up time of participants collected during and before the day of experimentation (D-1)

\begin{tabular}{lll}
\hline & $\begin{array}{ll}\text { Control group } \\
(\mathrm{n}=25)\end{array}$ & $\begin{array}{l}\text { IEI-EMF group } \\
(\mathrm{n}=30)\end{array}$ \\
\hline Bedtime & & \\
Mean & $23: 30$ & $22: 48$ \\
Width & $21: 30-01: 00$ & $21: 30-01: 00$ \\
Median & $23: 30$ & $22: 30$ \\
Waking time & & \\
Mean & $07: 29$ & $06: 23$ \\
Width & $06: 45-08: 20$ & $04: 00-08: 40$ \\
Median & $07: 30$ & $06: 45$ \\
\hline
\end{tabular}

